\title{
INFLUÊNCIA DO MÉTODO DE RECUPERAÇÃO OOCITÁRIA SOBRE OS PARÂMETROS QUANTI-QUALITATIVOS DE OÓCITOS BOVINOS
}

\author{
INFLUENCE OF THE RECOVERY METHOD ON THE QUANTI-QUALITATIVE PARAMETERS \\ OF BOVINE OOCYTES
}

\author{
M. V. O. SANTOS ${ }^{\text {; A. A. BORGES }}$; L. B. QUEIROZ NETA ${ }^{3}$; A. F. PEREIRA ${ }^{4}$
}

\begin{abstract}
RESUMO
Os objetivos foram avaliar os parâmetros quanti-qualitativos de oócitos bovinos após recuperação usando diferentes métodos de colheita e tipos de êmbolo da seringa de aspiração. Assim, dois experimentos foram realizados usando ovários de fêmeas post-mortem. No primeiro experimento, duas técnicas de colheita oocitária foram empregadas: aspiração de folículos $(2-8 \mathrm{~mm})$ com agulha $21 \mathrm{G}$ e seringa de $5 \mathrm{~mL}$ e, fatiamento da superfície ovariana (slicing). No segundo experimento, folículos $(2-8 \mathrm{~mm})$ foram aspirados usando seringa com distintos êmbolos (borracha $v s$. plástico). Os complexos cumulus-oócito (CCOs) foram classificados por critérios morfológicos em viáveis e não viáveis. Em seguida, CCOs foram corados com o azul de cresil brilhante (ACB) (60 min; $26 \mu \mathrm{M})$ e classificados como $\mathrm{ACB}^{+}$(viáveis) e $\mathrm{ACB}^{-}$(não viáveis). Um total de cinco repetições por experimento foi realizado e os dados analisados pelo teste exato de Fisher $(\mathrm{P}<0,05)$. No primeiro experimento, o número de oócitos por ovário obtido por slicing foi maior quando comparado à aspiração folicular $(14,8$ vs. 7,5; $\mathrm{P}<0,05)$. Contudo, um maior percentual de CCOs viáveis observado pelo ensaio de ACB foi obtido a partir da aspiração folicular $(65,7 \%$ vs. $31,0 \%$; $\mathrm{P}<0,05)$. No segundo experimento, nenhuma diferença $(\mathrm{P}>0,05)$ foi observada entre os tipos de êmbolos quanto aos parâmetros quantitativos. Quanto à qualidade oocitária avaliada por critérios morfológicos, um percentual maior de oócitos viáveis foi recuperado usando êmbolo de borracha $(75,4 \%$ vs. $58,2 \%$; $\mathrm{P}<0,05)$. Em conclusão, oócitos de melhor qualidade podem ser obtidos a partir da aspiração folicular, especialmente usando seringa com êmbolo de borracha.
\end{abstract}

PALAVRAS-CHAVE: Aspiração folicular. Azul de cresil brilhante. Colheita oocitária. Qualidade oocitária. Slicing.

\section{SUMMARY}

The aims were to evaluate qualitative-quantitative parameters of bovine oocytes after recovery using different collection methods and piston type from the aspiration syringe. Thus, two experiments were performed using ovaries from postmortem females. In the first experiment, two techniques of oocyte recovery were used: aspiration of follicles (2-8 mm) with $21 \mathrm{G}$ needle and $5 \mathrm{~mL}$ syringe, and slicing of the ovarian surface. In the second experiment, follicles (2-8 mm) were aspirated using syringes with different pistons (rubber vs. plastic). The cumulus-oocyte complexes (COCs) were classified by morphological criteria as viable and non-viable. Then, COCs were stained with brilliant cresyl blue (BCB) $(60 \mathrm{~min}, 26 \mu \mathrm{M})$ and categorized as $\mathrm{BCB}^{+}$(viable) and $\mathrm{BCB}^{-}$(non-viable). A total of five repetitions per experiment was performed and the data analyzed by Fisher's exact test $(\mathrm{P}<0.05)$. In the first experiment, the number of oocytes per ovary obtained by slicing was higher when compared to the follicular aspiration $(14.8 v s .7 .5 ; \mathrm{P}<0.05)$. Nevertheless, a greater percentage of viable COCs observed by BCB assay was obtained from the follicular aspiration $(65.7 \%$ vs. $31.0 \% ; \mathrm{P}<0.05)$. In the second experiment, no difference $(\mathrm{P}>0.05)$ was observed the piston type as the quantitative parameters. As the oocyte quality evaluated by morphological criteria, a higher percentage of viable oocytes were recovered using rubber piston $(75.4 \%$ vs. $58.2 \%$; $\mathrm{P}<0.05)$. In conclusion, better quality oocytes can be obtained from follicular aspiration, especially by using a rubber piston syringe.

KEY-WORDS: Follicular aspiration. Brilliant cresyl blue. Oocyte recovery. Oocyte quality. Slicing.

\footnotetext{
${ }^{1}$ Mestranda do Programa de Pós-Graduação em Ciência Animal, Universidade Federal Rural do Semi-Árido (UFERSA), Mossoró, RN, Brasil.

${ }^{2}$ Doutoranda do Programa de Pós-Graduação em Ciência Animal, UFERSA, Mossoró, RN, Brasil.

${ }^{3}$ Mestranda do Programa de Pós-Graduação em Ciência Animal, UFERSA, Mossoró, RN, Brasil.

${ }^{4}$ Docente do Centro de Ciências Biológicas e da Saúde, UFERSA, Mossoró, RN, Brasil. Autor para correspondência: alexsandra.pereira@ufersa.edu.br.
} 


\section{INTRODUÇÃO}

A produção in vitro de embriões (PIVE) possibilita vários benefícios para a reprodução animal em setores científicos, produtivos e tecnológicos (DE BEM et al., 2014). Essa biotécnica compreende várias etapas e cada uma tem influência direta no resultado final. De forma sucinta são as seguintes as etapas do processo: recuperação, seleção e maturação in vitro de complexos cumulus-oócito (CCOs), capacitação espermática seguida de fecundação in vitro, desenvolvimento in vitro dos presumíveis zigotos e transferência de embriões para receptoras sincronizadas (VARAGO et al., 2008). Em virtude de todas as suas aplicações, vários grupos têm buscado aumentar a eficiência da PIVE, visando conhecer os fatores envolvidos em cada etapa.

Uma vez que o desenvolvimento embrionário está intimamente relacionado à qualidade dos oócitos, estudos têm sido realizados a fim de aperfeiçoar os sistemas de colheita e avaliação qualitativa dos mesmos (IORIO et al., 2014; OPIELA; KATSKAKSIAZKIEWICZ, 2013). Em fêmeas post-mortem, a colheita oocitária é realizada pelas técnicas de aspiração folicular, utilizando agulha acoplada a seringa ou bomba de vácuo, e slicing, que consiste no fatiamento do córtex ovariano para exposição do líquido folicular (BERNAL et al., 2015). De modo geral, a aspiração folicular apresenta maior praticidade, além de permitir uma melhor seleção dos folículos que serão aspirados. Já o procedimento de slicing requer um maior tempo e apresenta um menor controle dos folículos que serão usados (SIANTURI et al., 2002). Essas características podem influenciar diretamente em dois aspectos importantes: a taxa de recuperação e a qualidade oocitária. Assim, ambas as técnicas podem apresentar vantagens e desvantagens; contudo, os dados com relação aos parâmetros citados ainda são controversos para bovinos (KAKKASSERY et al., 2010). Além disso, a composição dos materiais utilizados durante a colheita também pode diminuir a qualidade desses gametas, especialmente devido à toxicidade que podem apresentar para a reprodução (ENGLAND; ALLEN, 1992; NIJS et al., 2009).

Assim, a qualidade oocitária adquirida progressivamente durante a foliculogênese deve ser conservada durante a recuperação oocitária (SILVA et al., 2016). Portanto, os objetivos do presente trabalho foram avaliar os parâmetros quanti-qualitativos de oócitos bovinos após recuperação usando diferentes métodos de colheita e tipos de êmbolo da seringa de aspiração.

\section{MATERIAL E MÉTODOS}

Todos os experimentos foram realizados em concordância com Comitê de Ética de Uso de Animais (CEUA, no. 23091.001069/2015-79). Os reagentes usados foram adquiridos da Sigma-Aldrich (St. Louis, EUA) e quando necessário o pH das soluções e/ou meios foi ajustado para 7,4.

Dois experimentos foram realizados usando ovários de fêmeas bovinas adultas, mestiças e provenientes de abatedouro local. No primeiro experimento, duas técnicas de colheita oocitária foram empregadas: sistema de aspiração folicular constituído por seringa com êmbolo de borracha e agulha e fatiamento da superfície ovariana (slicing). No segundo experimento, folículos foram aspirados utilizando a mesma técnica de colheita com seringa, porém com dois tipos distintos de êmbolos (borracha vs. plástico).

Para tanto, os ovários foram transportados ao laboratório em solução salina $(\mathrm{NaCl}, 0,9 \%)$ aquecida a 35-37oC, e então lavados em uma nova solução de transporte e mantidos em banho-maria $(37 \mathrm{oC})$ para a colheita oocitária. Para a aspiração folicular, folículos de 2-8 mm de diâmetro foram aspirados com auxílio de uma agulha de $21 \mathrm{G}$ acoplada a uma seringa de $5 \mathrm{~mL}$ com êmbolo (borracha vs. plástico) contendo solução tampão fosfato (PBS). Já para o slicing, ovários foram alocados separadamente em placas de Petri e cortes foram realizados no córtex ovariano com distância de aproximadamente $2 \mathrm{~mm}$, utilizando uma lâmina de bisturi e priorizando as regiões com folículos de $2-8 \mathrm{~mm}$.

Após a colheita oocitária, o líquido folicular foi mantido em repouso por 15 min para sedimentação das estruturas. Decorrido esse tempo o conteúdo sedimentado foi examinado sob estereomicroscópio em aumento de 20x e 40x para a recuperação dos CCOs. Para classificação dos mesmos foram utilizados dois métodos: classificação morfológica convencional e ensaio de azul cresil brilhante (ACB). Assim, os oócitos foram classificados por critérios morfológicos em viáveis (pelo menos uma camada completa de células do cumulus e citoplasma homogêneo) e não viáveis (camada incompleta de células do cumulus e citoplasma homogêneo ou heterogêneo e/ou oócito degenerado), segundo Gonçalves et al. (2008).

Para o ensaio de ACB, os oócitos foram lavados em gotas de PBS e incubados com ACB diluído em PBS $(26 \mu \mathrm{M})$ por $60 \mathrm{~min}$ a 38,5 oC. Decorrido o tempo, os oócitos foram lavados novamente em PBS e classificados sob estereomicroscópio em $\mathrm{ACB}^{+}$ (viáveis; coloração azul no citoplasma) e $\mathrm{ACB}^{-}$(não viáveis; citoplasma incolor). $\mathrm{O}$ ensaio com o corante ACB permite avaliar a atividade da enzima glucose-6fosfato desidrogenase (G6PDH) que está relacionada ao metabolismo oocitário. Quando o oócito está em fase de crescimento a G6PDH apresenta elevada atividade, sendo capaz de reduzir o ACB de azul para incolor ( $\left.\mathrm{ACB}^{-}\right)$. Já em oócitos crescidos, a atividade da G6PDH é diminuída e o ACB não é reduzido fazendo com que os oócitos apresentem cor azul no citoplasma $\left(\mathrm{ACB}^{+}\right) \quad$ (OPIELA; KATSKA-KSIAZKIEWICZ, 2013). Assim, oócitos $\mathrm{ACB}^{+}$são considerados mais competentes para a PIVE e, portanto, classificados como viáveis.

Para análise dos dados, a taxa de recuperação oocitária (oócitos recuperados/ folículos aspirados), o número de oócitos colhidos por ovário e os percentuais de oócitos viáveis (oócitos viáveis/total de oócitos recuperados) por classificação morfológica e ensaio de ACB foram analisados pelo teste de exato de Fisher, usando o software Graphpad Instat 3.06 (GraphPad Software Inc., La Jolla, EUA). Para cada experimento, 
pelo menos cinco repetições foram realizadas. Os resultados foram expressos como média e/ou médias percentuais, sendo considerados significativamente diferentes quando $\mathrm{P}<0,05$.

\section{RESULTADOS E DISCUSSÃO}

No primeiro experimento, um total de 48 ovários foi utilizado (24 ovários/método de recuperação). Destes, 181 e 355 estruturas foram recuperadas por aspiração folicular e slicing, respectivamente. O número de oócitos por ovário obtido pela técnica de slicing foi maior quando comparado à aspiração folicular $(14,8 \mathrm{vs}$. 7,5; $\mathrm{P}<0,05)$. Diferença nesse parâmetro também foi observado por Wang et al. (2007) para oócitos bovinos (9,6 vs. 5,8). Em geral, a maior recuperação alcançada pela técnica de slicing pode estar associada ao procedimento que favorece a colheita de oócitos oriundos de folículos com pequeno diâmetro, os quais normalmente não são utilizados na aspiração folicular (SIANTURI et al., 2002). A seleção de folículos com diâmetro maior, até $8 \mathrm{~mm}$, durante a colheita favorece a obtenção de oócitos mais competentes, uma vez que folículos pequenos provavelmente não receberam estímulo suficiente de hormônios in vivo para $\mathrm{o}$ desenvolvimento posterior (DE BEM et al., 2014). Assim, o uso da técnica de slicing pode ser vantajoso quando se deseja uma alta taxa de recuperação e em casos de pouca disponibilidade de ovários para colheita.

Quanto à qualidade oocitária avaliada por critérios morfológicos (Tabela 1), nenhuma diferença foi observada entre as técnicas de recuperação $(\mathrm{P}>0,05)$. Sianturi et al. (2002), estudando esse mesmo parâmetro de avaliação oocitária, também não observaram influência dos métodos de recuperação sobre a qualidade de oócitos de bovinos oriundos de abatedouro (aspiração folicular: 27,71\%; slicing: 33,78\%). Já outros autores têm demostrado que CCOs de melhor qualidade podem ser obtidos a partir da técnica de slicing em comparação a aspiração folicular (CAROLAN et al., 1994; WANG et al., 2007). Nós acreditamos que seja necessário um impacto físico mais pronunciado para que ocorram alterações significativas na morfologia oocitária, o que não foi observado no presente trabalho e em outro estudo (SIANTURI et al., 2002) que comparou os sistemas de colheita.

Tabela 1 - Qualidade de oócitos bovinos obtidos por diferentes métodos de recuperação (aspiração folicular e slicing) e avaliados por critérios morfológicos e ensaio azul de cresil brilhante (ACB).

\begin{tabular}{|c|c|c|c|c|}
\hline \multirow{2}{*}{$\begin{array}{l}\text { Métodos de } \\
\text { recuperação oocitária }\end{array}$} & \multicolumn{2}{|c|}{ Análise morfológica } & \multicolumn{2}{|c|}{ Ensaio de ACB } \\
\hline & $\begin{array}{c}\text { Viáveis } \\
(\%)\end{array}$ & $\begin{array}{c}\text { Não viáveis } \\
(\%)\end{array}$ & $\begin{array}{c}\text { Viáveis } \\
(\%)\end{array}$ & $\begin{array}{c}\text { Não } \\
\text { viáveis }(\%)\end{array}$ \\
\hline Aspiração folicular & $\begin{array}{c}108 / 181 \\
(59,7)^{\mathrm{a}}\end{array}$ & $\begin{array}{c}73 / 181 \\
(40,3)^{\mathrm{a}}\end{array}$ & $\begin{array}{c}119 / 181 \\
(65,7)^{\mathrm{a}}\end{array}$ & $\begin{array}{l}62 / 181 \\
(34,2)^{\mathrm{a}}\end{array}$ \\
\hline Slicing & $\begin{array}{r}241 / 355 \\
(67,9)^{\mathrm{a}}\end{array}$ & $\begin{array}{r}114 / 355 \\
(32,1)^{\mathrm{a}}\end{array}$ & $\begin{array}{c}110 / 355 \\
(31,0)^{\mathrm{b}}\end{array}$ & $\begin{array}{c}245 / 355 \\
(69,0)^{\mathrm{b}}\end{array}$ \\
\hline
\end{tabular}

a,b : diferem na mesma coluna $(\mathrm{P}<0,05)$.

Em relação ao ensaio de $\mathrm{ACB}$, um maior percentual de CCOs viáveis $(\mathrm{P}<0,05)$ foi obtido a partir da aspiração folicular quando comparada ao slicing. Portanto, um maior percentual de CCOs não viáveis foi obtido a partir do slicing $(\mathrm{P}<0,05)$. Esse resultado pode estar relacionado à obtenção de CCOs mais desenvolvidos por meio da aspiração folicular, que permite a seleção mais eficiente dos folículos que serão utilizados. Na técnica de slicing, os cortes podem atingir regiões que apresentam folículos de pequeno diâmetro onde os oócitos não estão totalmente crescidos (FAIR et al., 1995; SIANTURI et al., 2002). Assim, é possível afirmar que para a comparação entre os métodos de colheita, o ensaio de ACB foi determinante para a avaliação eficiente da qualidade oocitária, uma vez que esse ensaio é capaz de identificar oócitos com crescimento finalizado e, portanto, mais aptos para as etapas posteriores da PIVE (OPIELA; KATSKA-KSIAZKIEWICZ, 2013).

Em geral, vários grupos têm utilizado o ensaio ACB a fim de auxiliar a avaliação morfológica convencional na classificação de oócitos de melhor qualidade e, consequentemente, aumentar o sucesso da PIVE (MIRSHAMSI et al., 2013; OPIELA; KATSKAKSIAZKIEWICZ, 2013). Normalmente, oócitos $\mathrm{ACB}^{+}$e com características morfológicas adequadas (considerados viáveis) possuem um melhor desempenho nas etapas de maturação e fecundação in vitro (MIRSHAMSI et al., 2013). Assim, alterações positivas ou negativas nos resultados de qualquer desses métodos de análise devem ser consideradas. No presente estudo, o ensaio de ACB foi importante para afirmar a aspiração folicular como método eficiente para maior recuperação de oócitos viáveis. A partir disso, outra avaliação no método de aspiração foi realizada, a qual consistiu na comparação dos tipos de êmbolo da seringa (borracha vs. plástico).

No segundo experimento, um total de 64 ovários (32 ovários /tipo de êmbolo) foi utilizado, perfazendo uma taxa de recuperação de 43,7\% (203/465) e 40,2\% (153/381) e número de oócitos/ovário de 6,3 e 5,1 para o êmbolo de borracha e plástico, respectivamente. Nenhuma diferença $(\mathrm{P}>0,05)$ foi observada entre os tipos de êmbolos quanto aos parâmetros quantitativos.

Quanto à qualidade oocitária avaliada por critérios morfológicos, um percentual maior de oócitos viáveis foi recuperado usando êmbolo de borracha (Tabela 2). Contudo, nenhuma diferença foi observada na qualidade de oócitos avaliada pelo ensaio de ACB ( $\mathrm{P}>0,05$, Tabela 2) para êmbolo de borracha e plástico Assim, apesar das seringas com êmbolo de plástico serem inertes, evitando possíveis reações com o fluido folicular, a qualidade dos oócitos foi afetada positivamente pelo êmbolo de borracha, utilizando a 
morfologia como método de análise da qualidade oocitária. Tal resultado pode ser atribuído a maior rigidez do êmbolo de plástico, o qual pode ter causado um desprendimento maior das células do cumulus, promovendo a diminuição no percentual de oócitos morfologicamente viáveis. Contudo, Iorio et al. (2014), usando êmbolo de borracha para a obtenção de estruturas ovinas para a fecundação in vitro, verificaram que com $96 \mathrm{~h}$ de cultivo in vitro, o desenvolvimento embrionário foi interrompido, apresentando sinais de degeneração. Essa interrupção pode estar relacionada com o látex encontrado na seringa, o qual pode reagir com o fluido folicular e talvez interferir no desenvolvimento embrionário e, que nos primeiros momentos de recuperação oocitária não gera danos na qualidade das estruturas.

Estudos anteriores demonstraram que seringas com êmbolo de borracha apresentam efeitos tóxicos em testes utilizando espermatozoides (CRITCHLOW et al., 1989; CLAASSENS et al., 2000). Contudo neste estudo, utilizando o ensaio de ACB, a qualidade de oócitos imaturos não foi afetada pela composição da seringa. Provavelmente o curto tempo de exposição dos oócitos a borracha do êmbolo não foi capaz de estabelecer o efeito tóxico em relação à seringa sem borracha (ENGLAND; ALLEN, 1992).

Tabela 2 - Qualidade de oócitos bovinos obtidos por diferentes êmbolos de seringa de aspiração folicular (borracha $v s$. plástico) e avaliados por critérios morfológicos e ensaio de azul de cresil brilhante (ACB).

\begin{tabular}{ccccc}
\hline \multirow{2}{*}{ Tipo de êmbolo } & \multicolumn{2}{c}{ Análise morfológica } & \multicolumn{2}{c}{ Ensaio de ACB } \\
& Viáveis $(\%)$ & Não viáveis $(\%)$ & Viáveis $(\%)$ & Não viáveis $(\%)$ \\
\cline { 2 - 5 } Borracha & $153(75,4)^{\mathrm{a}}$ & $50(24,6)^{\mathrm{a}}$ & $134(66,0)^{\mathrm{a}}$ & $69(34,0)^{\mathrm{a}}$ \\
Plástico & $89(58,2)^{\mathrm{b}}$ & $64(41,8)^{\mathrm{b}}$ & $92(60,1)^{\mathrm{a}}$ & $61(39,9)^{\mathrm{a}}$ \\
\hline
\end{tabular}

$\overline{\mathrm{a}, \mathrm{b}}$ : diferem na mesma coluna $(\mathrm{P}<0,05)$.

\section{CONCLUSÃO}

Ambos os métodos (aspiração folicular e slicing) podem ser utilizados para recuperação de oócitos bovinos oriundos de fêmeas post-mortem. Contudo, apesar da maior recuperação oocitária através da técnica de slicing, o ensaio de azul cresil brilhante mostrou que oócitos oriundos de aspiração folicular possuem uma melhor qualidade, parâmetro importante para a determinação da competência oocitária. Além disso, o tipo de êmbolo da seringa não afetou a quantidade de oócitos recuperados; contudo, a aspiração folicular usando êmbolo de borracha resultou em um número maior de oócitos viáveis de acordo com a avaliação morfológica.

\section{AGRADECIMENTOS}

Este estudo foi apoiado pelo Conselho Nacional de Desenvolvimento Tecnológico e Científico (CNPq, processo no. 477710/2013-1).

\section{REFERENCIAS}

BERNAL, S.M.; HEINZMANN, J.; HERRMANN, D.; TIMMERMANN, B.; BAULAIN, U.; GROßFELD, R.; DIEDERICHA, M.; LUCAS-HAHNA, A.; NIEMANN, H. Effects of different oocyte retrieval and in vitro maturation systems on bovine embryo development and quality. Zygote. Cambridge. v.23, p.367-377, 2015.

CAROLAN, C.; MONAGHAN, P.; GALLAGHER, M.; GORDON, I. Effect of recovery method on yield of bovine oocytes per ovary and their developmental competence after maturation, fertilization and culture in vitro. Theriogenology. New York. v.41, p.10611068, 1994.
CLAASSENS, O.E.; WEHR, J.B.; HARRISON, K.L. Optimizing sensitivity of the human sperm motility assay for embryo toxicity testing. Human Reproduction. Oxford. v.15, p.1586-1591, 2000.

CRITCHLOW, J.D.; MATSON, P.L.; MAUREEN, C.N.; HORNE, G.; TROUP, S.A.; LIEBERMAN, B.A. Quality control in an in vitro fertilization laboratory: use of human sperm survival studies. Human Reproduction. Oxford. v.4, p.545-549, 1989.

DE BEM, T.H.C.; ADONA, P.R.; BRESSAN, F.F.; MESQUITA, L.G.; CHIARATTI, M.R.; MEIRELLES, F.V.; LEAL, C.L.V. The influence of morphology, follicle size and Bcl-2 and Bax transcripts on the developmental competence of bovine oocytes. Reproduction in Domestic Animals. Linköping. v.49, p.576-583, 2014.

ENGLAND, G.C.W.; ALLEN, W.E. Factors affecting the viability of canine spermatozoa: I. Potential influences during processing for artificial insemination. Theriogenology. New York. v.37, p.363-371, 1992.

FAIR, T.; HYTTEL, P.; GREVE, T. Bovine oocyte diameter in relation to maturational competence and transcriptional activity. Molecular Reproduction and Development. Linköping. v.42, p.437-442, 1995.

GONÇALVES, P.B.D.; OLIVEIRA, M.A.L.; MEZZALIRA, A.; MONTAGNER, M.M.; VISINTIN, J.A.; COSTA, L.F.S. Produção in vitro de embriões. In: GONÇALVES, P.B.D.; FIGUEIREDO, J.R.; FREITAS, V.J.F. (Eds.). Biotécnicas Aplicadas à Reprodução Animal. São Paulo: Roca, p. 261-291, 2008.

IORIO, G.A.; PUMARÁ, P.D.; RAFAELLI, P.M. Influence of rubber piston syringe used in recovery of oocytes in the development of sheep embryos produced in vitro. Animal Reproduction. Belo Horizonte. v.11, p.420, 2014. 
KAKKASSERY， M.P.; VIJAYAKUMARAN, V.; SREEKUMARAN, T. Effect of cumulus oocyte complex morphology on in vitro maturation of bovine oocytes. Journal of Veterinary and Animal Sciences. New York. v.41, p.12-17, 2010.

MIRSHAMSI, S.M.; KARAMISHABANKAREH, H.; AHMADI-HAMEDANI, M.; SOLTANI, L.; HAJARIAN, H.; ABDOLMOHAMMADI, A.R. Combination of oocyte and zygote selection by brilliant cresyl blue (BCB) test enhanced prediction of developmental potential to the blastocyst in cattle. Animal Reproduction Science. Amsterdam. v.136, p.245-251, 2013.

NIJS, M.; FRANSSEN, K.; COX, A.; WISSMANN, D.; RUIS, H.; OMBELET, W. Reprotoxicity of intrauterine insemination and in vitro fertilizationembryo transfer disposables and products: a 4-year survey. Fertility and Sterility. New York, v.92, p.527$535,2009$.

OPIELA， J.; KĄTSKA-KSIĄŻKIEWICZ， L. The utility of brilliant cresyl blue (BCB) staining of mammalian oocytes used for in vitro embryo production (IVP). Reproductive Biology. New York. v.13, p.177-183, 2013.
SIANTURI, R.G.; THEIN, M.; WAHID, H.; ROSNINA, Y.C. Effect of collection technique on yield of bovine oocytes and the development potential of oocytes from different grades of oocytes. Indonesian Journal of Animal and Veterinary Sciences. v.7, p.188193, 2002.

SILVA, J.R.V.; VAN DEN HURK, R.; FIGUEIREDO, J. R. Ovarian follicle development in vitro and oocyte competence: advances and challenges for farm animals. Domestic Animal Endocrinology. Amsterdam. v.55, p.123-135, 2016.

VARAGO, F.C.; MENDONÇA, L.F.; LAGARES, M.A. Produção in vitro de embriões bovinos: estado da arte e perspectiva de uma técnica em constante evolução. Revista Brasileira de Reprodução Animal. Belo Horizonte. v. 36, p. 100-109, 2008.

WANG, Z.G.; YU, S.D.; XU, Z.R. Effects of collection methods on recovery efficiency, maturation rate and subsequent embryonic developmental competence of oocytes in Holstein cow. Asian Australasian Journal of Animal Sciences. Seoul. v.20, p.496-500, 2007. 